\title{
Precarización del trabajo y estrategias de trabajadoras en plataformas digitales: trabajo descle el hogar, organización sindical y disputa por derechos en el contexto de la pandemia del Covid-19
}

\section{Sofía Scasserra* Flora Partenio*}

\section{Resumen}

El presente artículo indaga en las condiciones de mujeres que se fueron insertando en trabajos más precarios, producto de la falta de respuestas en materia de políticas de corresponsabilidad de los cuidados. Estas trabajadoras buscaron respuestas en el trabajo remoto y en las plataformas de trabajo. La sobrecarga de trabajo doméstico y de cuidados no remunerados limita las posibilidades de inserción en empleos con jornadas laborales extradomésticas. De esta manera, la inserción de mujeres en plataformas de trabajo menos visibles - por no estar "en la calle" - es notoria porque habilita la combinación de tiempos y tareas - remuneradas y no remuneradas - en el hogar, pero, al mismo tiempo, las encasilla en sectores que les fueran socialmente asignados. Esta realidad requiere de nuevas estrategias políticas y sindicales a fin de darles visibilidad y representatividad en las organizaciones. Se materializa el riesgo de generar sindicatos sin la participación de las mujeres y sin una agenda de género. El artículo retoma las implicancias de un debate histórico que aún sigue vigente, y que se ha evidenciado en el contexto de la pandemia global, en torno a la llamada crisis de los cuidados. La pandemia del Covid-19 aceleró e

* Universidad Nacional de Tres de Febrero, Tres de Febrero, BA, Argentina.

** Universidad Nacional Arturo Jauretche, Florencia Varela, BA, Argentina. 
hizo más vigente que nunca estos debates. Basado en un enfoque cualitativo, el presente estudio recurre a entrevistas semiestructuradas y al análisis documental para analizar las condiciones laborales de trabajadoras en plataformas digitales para el caso argentino. Asimismo, basado en una serie de talleres con trabajadoras sindicalizadas, plantea diversas estrategias para una agenda sindical y feminista, a fin de que no se diluyan la participación y derechos de las mujeres y personas LGBT + a través de plataformas de trabajo que las aíslen en diversos empleos bajo la modalidad virtual y remota.

Palabras clave: trabajo, capitalismo de plataformas, mujeres, precarización, sindicatos.

\section{Labor precariousness and strategies of women working on digital platforms: work from home, union organization and struggle for rights in the context of the Covid-19 pandemic}

\section{Abstract}

The present work seeks to investigate the conditions of women workers who have entered the labor market in more precarious jobs as a result of the lack of policies to address co-responsibility for care work. These workers have resorted to remote jobs and work at digital platforms for being overloaded with unpaid care work, what limits their possibilities of entering jobs with extra-domestic working hours. So, women's presence in less visible work platforms - for not being "on the street" - is evident. While allowing them to accommodate time and (paid and unpaid) tasks, it also places them in sectors that were socially assigned to them, thus reinforcing stereotypes and overloading their workdays. This reality requires new political and union strategies to render them visible and represented within institutions. There is a risk of emptying unions of women's participation and of a gender agenda. The article takes up again the implications of a historical and ongoing debate that has become evident in the context of the global pandemic, regarding the so-called care crisis. The Covid 19 pandemic accelerated and made these debates more relevant than ever. The search for solutions is urgent, and this work proposes various strategies in the case of Argentina that should be promoted by unions, social and feminists' movements, in order not to undermine the participation and rights of women \& LGBT+ people, through digital platforms that isolate them in various virtual and remote jobs.

Key words: labour, platform capitalism, women, precariousness, trade unions 


\section{Introducción}

on la aparición y expansión de las nuevas tecnologías de información
y comunicación (TICs), el trabajo flexible y mediado por estas
tecnologías se ha vuelto una realidad cada vez más palpable y extendida en las modalidades laborales. Es posible advertir trabajadores de todos los sectores que utilizan su celular o su computadora como herramienta y lugar de trabajo, estén donde estén. Cada vez son más personas las que encuentran en la economía de plataformas una oportunidad de generar ingresos o complementarlos, ya sea trabajando desde su casa o combinando horarios en una "jornada flexible" con otros empleos remunerados. Con la pandemia del Covid-19, dichas modalidades - que ya se encontraban presentes en diferentes ámbitos - se extendieron masivamente frente a la imposibilidad de las personas de trasladarse y moverse de sus hogares. Estas modalidades se combinaron con la extensión del teletrabajo para quienes ya se encontraban en relación de dependencia y, debido a las medidas de confinamiento y/o aislamiento social, debieron cumplir con sus tareas desde sus hogares (por ejemplo, docentes de todos los niveles educativos, sector servicios, administración, estatales, entre otros).

De acuerdo a los estudios previos a la pandemia del Covid-19 (OIT, 2019), las plataformas de trabajo dedicadas a la asignación de microtareas se habían convertido en una opción para mujeres de distintas regiones, ya que les permitía la posibilidad de combinar la carga de trabajo no remunerado con las actividades remuneradas. Principalmente en el caso de los países del Sur global, sin haber resuelto aún la división sexual del trabajo en los hogares y sin contar con políticas de corresponsabilidad de los cuidados, la modalidad de empleos remotos y trabajo vía plataformas se volvió una oportunidad para mujeres que asumen la mayor carga de trabajo doméstico y de cuidados no remunerado. Bajo el contexto de la pandemia, en el marco de medidas de confinamiento y aislamiento social ${ }^{1}$ decretadas por

1 Al respecto, ver para el caso argentino el Decreto 297/2020 del Poder Ejecutivo con fecha 19/03/2020: https://www.boletinoficial.gob.ar/detalleAviso/primera/227042/20200320 
los gobiernos, esta sobrecarga de trabajo no remunerado puso en evidencia de modo más crudo la crisis de los cuidados en América Latina y el Caribe (CEPAL, 2020). Esta crisis se combina con una caída general en los ingresos, con la imposibilidad de generar ingresos en el marco del confinamiento a través de trabajos informales, con la destrucción de puestos de trabajo, el aumento del desempleo para las mujeres y personas LGBT+.

Estas modalidades de trabajo vía plataformas digitales conllevan una serie de desafíos a los derechos laborales, la seguridad y protección sociales: trabajadores que mueven la economía digital y, en especial, trabajadoras se insertan en empleos cada vez más precarios sin jubilación, sin licencias, sin condiciones y medio ambiente de trabajo adecuadas, sin salario mínimo ni vacaciones (Partenio, 2020a). Asimismo, la sindicalización se dificulta tanto en las modalidades de trabajo remoto como en las enmarcadas en plataformas de trabajo, cuando se trata de tejer solidaridades y construir demandas con quienes trabajan desde su casa, conectadas a través del algoritmo de una red social o un medio de comunicación masivo. Estos desafíos tienen que ser sorteados por las organizaciones sociales, sindicales y feministas para que no operen de manera encubierta en contra de todos los derechos que se vienen ganando en los últimos años. Pensando en el caso de Argentina, resulta clave garantizar los derechos que ya están asentados en la Constitución Nacional y en las convenciones colectivas de trabajo y, al mismo tiempo, visualizar en que áreas o sectores se debe avanzar con la regulación en materia laboral.

Ante los desafíos que enfrentan las formas de organización de les trabajadores frente al avance de la digitalización y el trabajo en plataformas, este artículo se concentrará principalmente en las plataformas de trabajo para el caso argentino, desde la declaración del Aislamiento Social, Preventivo y Obligatorio (ASPO) decretado por el poder ejecutivo a mediados de marzo de 2020. En este sentido, entendemos que se abre una serie desafíos para diseñar estrategias de organización frente a las modalidades de precarización que habilitan estas formas de trabajo. Entre ellos, las dinámicas laborales que exigen a trabajadores estar disponibles las $24 \mathrm{hs}$., los 7 días de la 
semana y los 365 días del año. En materia de analizar las nuevas formas de precarización que puedan generarse para las mujeres y personas LGBT+, resulta clave la articulación de la agenda feminista con los sindicatos. Asimismo, resulta pertinente para este análisis considerar los alcances de estas modalidades, frente a la incorporación creciente de las trabajadoras en puestos de trabajo mediados por las nuevas tecnologías ${ }^{2}$ que permiten resituar las tareas y responsabilidades laborales en los hogares, donde se vuelven a poner en tensión las esferas de la producción y la reproducción.

En términos metodológicos, este estudio se enmarca en una perspectiva cualitativa, recurriendo a tres estrategias de recolección de información. Se realizaron entrevistas a informantes clave referentes de plataformas web y entrevistas semiestructuradas con trabajadoras enmarcadas en plataformas de trabajo. En el caso de las entrevistas con trabajadoras, se han realizado con vendedoras online, sector bancario y de microtareas. En el caso de las plataformas, se han realizado entrevistas con responsables y encargados de áreas en Facebook y Mercado Libre en Buenos Aires. También se ha recurrido al análisis documental de materiales emitidos por empresas de plataformas, organizaciones sindicales, y proyectos de ley que intentaron regular estas modalidades previamente y durante la pandemia. Se analizaron comunicados de organizaciones sindicales, convenios colectivos, cuadernillos de formación, materiales de las áreas de género etc. Finalmente, se ha realizado observación - en el contexto previo a la pandemia - en talleres de formación sindical.

El artículo se encuentra organizado en cuatro apartados. En primer lugar, se retoma la importancia que han cobrado los debates provenientes de los activismos y estudios feministas alrededor de la organización social del cuidado. Se trata precisamente de la dimensión e importancia que adquiere el trabajo doméstico y de cuidados no remunerado cuando se discute sobre

${ }^{2}$ En este caso puede abarcar una diversidad de herramientas, condiciones e innovaciones tecnológicas: dispositivos que garantizan el acceso a la conectividad; manejo de portales de internet; análisis y procesamiento de información; manejo de la comunicación; formación a distancia a través de campus virtuales; conocimiento y manejo de la infraestructura de la economía digital, IA etc. 
la extensión de la virtualización y el trabajo vía plataformas en la pandemia del Covid-19. En un segundo apartado se presenta un mapeo de la presencia de trabajadoras en las plataformas web, marcando las diferencias, tipos y contextos. En el tercer apartado se explora la disputa que se ha dado en Argentina por los derechos en el marco de la pandemia. Finalmente, el último apartado presenta una serie de implicancias y estrategias posibles para la organización sindical, en particular, en torno las modalidades enmarcadas en el teletrabajo y en la discusión por la regulación de las plataformas.

\section{La crisis de los cuidados en un "futuro del trabajo" que ya llegó}

Los interrogantes en torno al "futuro del trabajo" en el marco de las nuevas tecnologías de la información y comunicación reinstalaron un viejo tópico que había moldeado el debate sobre el nudo producción/ reproducción en el movimiento feminista. Sin embargo, con la extensión de la pandemia del Covid-19, estos efectos se han acelerado, ya que el confinamiento y el aislamiento social, con cierre de escuelas y espacios educativos, ha puesto en evidencia la crisis de los cuidados y la sobrecarga de las mujeres con estas tareas (Partenio, 2020b). Frente a las modalidades generadas por el capitalismo de plataformas, cabe preguntarse por la fuerza que cobran estos planteos - enarbolados por los feminismos en la década del '70 - y bajo que nuevas aristas son revisitados a partir de las implicancias de la economía del cuidado.

Fue así como durante la década del setenta se provocaron debates en el Norte y en el Sur global - donde se evidenció lo que algunas autoras llamaron el "infeliz matrimonio entre marxismo y feminismo", debido a que las reivindicaciones feministas volvían a quedar subordinadas en los análisis y estrategias políticas, entre ellas, la valoración de las tareas que permitían la reproducción de la fuerza de trabajo. Estos intercambios de posiciones cobraron forma en el conocido "debate sobre el trabajo doméstico" (Rodríguez; Cooper, 2005). Y justamente porque la organización 
social del cuidado continúa siendo injusta en el mundo, especialmente en los países del Sur global, es que estos debates han sido reactualizados.

En los últimos años, los debates en torno al "futuro del trabajo" y la industria 4.0 habían permeado las preocupaciones de organismos supranacionales, de las organizaciones sindicales, y de los estudios laborales. La primera cuestión a dilucidar es iqué se entiende por revolución 4.0? Se trata del avance creciente de la automatización, la robotización y la inteligencia artificial que dan forma a la llamada "nueva revolución tecnológica", la cual pone en riesgo los puestos de trabajo en sectores manufactureros y en actividades de servicios caracterizadas por su alta feminización y/o de baja calificación (Vaca Trigo, 2019). Se trata, también, de la organización de trabajadores y trabajadoras, por medio de algoritmos (Scasserra, 2019) que procesan datos obtenidos de la automatización y digitalización del empleo (Scasserra; Sai, 2020), en un nuevo capitalismo de vigilancia (Zuboff, 2019). Este capitalismo cibernético (Sai, 2019) es entendido como una forma más eficiente de capital que va subsumiendo las formas tradicionales de capital industrial y financiero y las traduce en su versión cibernética, utilizando la implementación de tecnología para el fraude laboral (Ottaviano, 2020).

En un escenario de pandemia global, donde se han exacerbado las desigualdades, se abren al menos dos interrogantes en torno a las condiciones de expansión de este capitalismo cibernético ¿cómo se incorporan las trabajadoras en estas plataformas de trabajo?, ¿cuáles son las razones por las que optan por estas modalidades?, ¿qué vigencia cobran los debates en torno al trabajo doméstico y de cuidados no remunerados? $\mathrm{Si}$, en los '70, estas lecturas feministas interpelaban aquellas miradas cuya expectativa estaba puesta en la emancipación de las mujeres a partir de la incorporación en el trabajo asalariado, ¿cómo es posible analizar, en pleno siglo XXI, las condiciones bajo las cuales las trabajadoras optan por el empleo de plataformas? ¿qué nuevas formas de precariedad se expanden?

En el "debate sobre el trabajo doméstico" que se dio en los '70, se explicitó el intento de convertirlo en un objeto específico de análisis 
recurriendo a los marcos analíticos marxistas y materialistas. Estos análisis se desarrollaron en distintas vertientes tales como las francófonas, inglesas, norteamericanas, italianas y latinoamericanas (Partenio, 2013). También se expresaron una serie de controversias al interior del propio feminismo, por ejemplo, con las posturas del feminismo radical desde las cuales se consideraba la relación de los sexos como el antagonismo social principal. Veamos en detalle cómo fue considerado y valorizado el trabajo de las mujeres por estas corrientes que eran parte de un activo movimiento feminista.

En cada una de las vertientes de los feminismos socialistas y materialistas se buscó ubicar al "sexo" en un eje específico de opresión, reelaborando y ampliando categorías marxistas, o bien, proponiendo nuevas. En ese debate, algunas reelaboraron las categorías de producción-reproducción y el concepto de "trabajo", mientras que otras ampliaron la realidad de la base material y de los ámbitos de explotación que excedían los planteos meramente económicos. Desde Italia, Mariarosa Dalla Costa, proveniente del obrerismo italiano, focaliza su análisis en la reproducción de la fuerza de trabajo, considerando que el trabajo doméstico constituía el corazón del problema y era la palanca de poder de las mujeres contra el capitalismo. Los diálogos entre Dalla Costa y Silvia Federici, sumado a las articulaciones con otras feministas del Norte como Selma James, dieron lugar a potentes estrategias y campañas de internacionalización del debate y por la valorización del trabajo doméstico durante los '70 (Partenio, 2013). Desde su análisis, se insistía en señalar el disciplinamiento de las mujeres al salario masculino, que había dado lugar a un proceso de expansión del "patriarcado del salario" (Federici, 2018a). A partir del "trabajo vital" de las mujeres como "operarias de la casa" estaba "en nuestras manos" una formidable "palanca de poder", ya que podían "rechazar producir" y, a partir de ahí, exigir un nuevo tipo de desarrollo que garantizara nuevas condiciones - en primer lugar, la "autonomía económica" de ellas y el reparto equitativo del trabajo doméstico con los varones (Dalla Costa, 2009).

En medio de las reformas estructurales que el neoliberalismo imponía en la década del '80 en los países del Norte y luego en países del Sur, Federici 
(2018b) consideró que uno de los desaciertos más grandes del feminismo fue apostar por la emancipación esperada a partir de la incorporación masiva en el mercado laboral y en las nuevas formas de empleo, que termina por consumir la vida de las mujeres en extensas jornadas laborales y sin generar autonomía económica. En ese rumbo, el feminismo se ha fragmentado por separar el trabajo doméstico del resto de las actividades profesionales y laborales, y porque abandona la discusión sobre quien asume la carga de trabajo no remunerado en los hogares. Posteriormente, un sector de economistas feministas se comienza a señalar los costos que tiene la expansión de la globalización para las mujeres, tanto en el Norte como en el Sur (Villota, 2001).

Los debates sobre el trabajo doméstico se vieron revitalizados a partir de los estudios económicos enfocados en la definición de una crisis de la reproducción social (Picchio, 1992; Carrasco, 1999). La contribución desde el campo de la Economía Feminista (EF) como parte de las corrientes heterodoxas de la economía ha sido la revitalización de la discusión del nudo producción-reproducción, a través de la propuesta de herramientas conceptuales que permitan caracterizar la economía del cuidado. Desde este campo de estudios se visibiliza el rol sistémico del trabajo doméstico y de cuidados no remunerados como un vector de desigualdad que garantiza la reproducción cotidiana de la vida y, por ende, de la propia fuerza de trabajo. Parte de la tarea de la EF ha sido analizar la injusta organización social del cuidado que modela las sociedades, identificando los elementos que reproducen las desigualdades de género. En el caso de Argentina, se ha señalado la ausencia de políticas de corresponsabilidad y de un sistema integrado de cuidados que permita resolver la sobrecarga de trabajo que recae sobre las mujeres (Rodríguez Enríquez; Marzonetto, 2015).

En plena pandemia global del Covid-19, la extensión de modalidades de empleo remoto y de plataformas digitales se presenta como forma de "conciliación" con el trabajo doméstico y de cuidados no remunerado que recae mayoritariamente sobre las mujeres. Considerando que hay países que se encuentran aún con medidas de aislamiento o distanciamiento 
social, que les impiden a las personas concurrir a sus lugares de trabajo, ¿qué significa en plena pandemia pensar la "conciliación" entre trabajo remunerado desde el hogar y trabajo de cuidados no remunerado? A su vez, la inserción de mujeres en plataformas digitales hace pensar que la flexibilidad de horarios condice con la conciliación antedicha. ¿Qué diferencia - en materia de "conciliación" - establece para las mujeres el hecho de trabajar en un empleo remoto ${ }^{3}$, en una plataforma de trabajo o en generar ingresos a través de plataformas digitales que no son de trabajo?

\section{Trabajadoras en las plataformas digitales: $y$ antes de la pandemia, ¿qué?}

Desde los foros económicos y los organismos internacionales - tales como la OMC, el G20, el W20 -, se ha pregonado la inclusión digital de las mujeres como una llave para inclusión económica y el desarrollo. En estos discursos, se presenta a la tecnología como neutral al género y, por ende, como con capacidad para igualar condiciones. En las reuniones de las ministeriales de la Organización Mundial de Comercio (2019), se han utilizado estos discursos para impulsar acuerdos sobre comercio electrónico que intentan desregular el mercado de datos y garantizar la expansión de la economía digital.

Estos discursos se apoyan en argumentos centrados en enfatizar el poder igualador de internet donde el acceso, la posibilidad de ofertar servicios y la remuneración es la misma para todas las personas, sin importar su género, edad, orientación sexual, raza, clase o lugar de residencia. Este señalamiento se apoya en el hecho de garantizar que quien está del otro lado de la pantalla desconoce a quién está ofreciendo sus servicios profesionales o

${ }^{3}$ El empleo remoto comprende aquellos trabajos que están facilitados por el uso de tecnología y es posible encontrar que, en algunos casos, las empresas combinan la presencia física en el lugar de trabajo con las jornadas "flexibles" desarrolladas en los hogares de trabajadores. Este trabajo puede - o no - implicar el uso de plataformas digitales. En el contexto de pandemia estas modalidades se extendieron a distintos sectores. En términos normativos, en países como Argentina se multiplicaron los debates y la presentación de proyectos para regular el "teletrabajo" (Scasserra, 2020). 
vendiendo de manera online un producto. Este argumento a favor de las políticas de inclusión digital desconoce la existencia de brechas digitales de género. Estas brechas se acrecientan si se incorpora una mirada del mundo del trabajo que no sea heteronormativa y binaria ${ }^{4}$.

Estos discursos hegemónicos se ponen en tensión cuando se analiza el acceso e inserción laboral de las mujeres y personas trans en plataformas de trabajo. Dicha inserción se encuentra atravesada por la intersección de desigualdades de clase, raza/etnia, género, edad y orientación sexual que se articulan con las condiciones de acceso y permanencia en el mercado laboral. Estas desigualdades se han profundizado durante el largo año de pandemia por Covid-19, ya que se han evidenciado las diferencias en el acceso a la conectividad, la ausencia de redes de cuidados y las formas de violencias contra las mujeres y personas LGBT + en contextos de cuarentana o confinamiento. En este sentido, resulta clave hacer una categorización de las formas de inserción de las trabajadoras en la economía digital, para analizar en detalle las opciones, oportunidades y condiciones laborales generadas por las distintas modalidades de trabajo remoto, sean estos de plataformas o no.

\section{Un mapeo de la presencia de trabajadoras en las plataformas}

Cuando se habla de "economía de plataformas" se suele asociar a los conceptos de gig-economy, on-demand economy, crowdsourcing, microworkers, collaborative economy y uberización del empleo. Sin embargo, la heterogeneidad de las tareas e intercambios realizados a través de estas plataformas ha generado diferencias en las formas de situar el trabajo, la calificación laboral, la construcción de perfiles de usuarios-consumidores

\footnotetext{
${ }^{4}$ En este punto resultan claves los relevamientos de los colectivos LGBT+ sobre las brechas digitales y las dinámicas de exclusión que padecen las personas trans y travestis del mundo laboral. De acuerdo al análisis de ATTTA (2020) en Argentina menos del 10\% de la población trans logra acceder a un empleo y, en muchos casos, las personas trans no cuentan con las herramientas digitales y dispositivos para generar su primer currículum vitae o subirlo a una plataforma.
} 
y usuarios-proveedores, la remuneración, la disponibilidad de tiempo, la evaluación de desempeño etc. (Partenio, 2020a). Se trata de una nueva forma de organizar los mercados donde internet conecta y las plataformas son el lugar de búsqueda de bienes y servicios para que la oferta se adecue a la demanda. Pero ¿quién provee la oferta? Desde los discursos de la "economía colaborativa" se habla de "emprendedores". Las críticas a esta denominación de la economía ya fueron enunciadas (Slee, 2016) y se ha resaltado - para el caso argentino - la flexibilidad y el ocultamiento de las relaciones laborales (Del Bono, 2020).

Sin embargo, la figura del "emprendedurismo" quedó bien lejos en las plataformas de trabajo, donde la estrategia, el diseño, el talento y la capacidad de negocios, poco tienen que ver con el grado de control que las personas dichas emprendedoras tienen sobre sus condiciones de trabajo y su nivel de ingreso. En efecto, las plataformas fijan y parametrizan todo: las condiciones de pago, las formas y tiempos de entrega, los descuentos y promociones, los niveles de exposición y publicidad (basados en sistemas de calificación algorítmica), los tiempos de trabajo y la necesidad de disponibilidad. En diferentes plataformas del mundo se comenzaron a utilizar los llamados "contratos de hora cero", y el empleo de plataforma es la expresión virtual de ese tipo de contratos, donde trabajadores deben estar disponibles por tiempo indeterminado y se les paga solo cuando su servicio es requerido. Las desigualdades en el eje Norte-Sur muestran las disparidades entre la oferta y demanda de trabajo en plataformas y, a la vez, explicitan las diferencias de remuneraciones entre las mismas regiones del sur-global (OIT, 2019). Asimismo, es en este sector donde las brechas y desigualdades de género se ensanchan en diversos ángulos. En algunas plataformas, puede incidir y reforzar la precariedad la condición migratoria. De acuerdo al mapeo realizado para el caso argentino y en diálogo con los estudios recientes, es posible destacar los siguientes puntos:

- Asimetrías en la remuneración de varones y mujeres. De acuerdo a estudios precedentes, trabajadoras y trabajadores no ganan la misma 
remuneración por el mismo servicio brindado (Hawkins, 2018; Partenio 2020a). Eso se debe principalmente a la discriminación que sufren las mujeres en internet. Un estudio reciente en países del Norte (Cook et al., 2020) demuestra que las choferas de Uber ganan un $7 \%$ menos que sus colegas varones debido a la discriminación que sufren por parte de los pasajeros a la hora de calificarlas (Hawkins, 2018; Gaskell, 2018).

- El algoritmo como jefe. La algoritmización de las relaciones laborales Ileva a que cada trabajadora o trabajador sea calificado constantemente por un sistema automatizado de inteligencia artificial. No hay intermediarios humanos, no hay excusas ni justificaciones que puedan presentarse a la "vieja" oficina de personal (Scasserra, 2019). Y las mujeres, una vez más, son las que más sufren en esta situación por las formas de segregación y brechas que se imponen. Al hacerse cargo del trabajo doméstico y de cuidados no remunerado, a las mujeres se les hace más difícil satisfacer las demandas del algoritmo de calificación y gestión laboral, desprovistas de derechos, a diferencia del desempeño de sus colegas varones. Los niveles de performance entre los géneros varían en el empleo de plataforma y esto se debe principalmente a la sobrecarga de trabajo no remunerado de la mujer (OIT, 2019).

- Las plataformas de trabajo han reforzado los estereotipos de género. Las estadísticas para el caso argentino son reveladoras: las mujeres se insertan en plataformas que tienen más que ver con la economía del cuidado. El informe del CIPPEC (Madariaga et al., 2019) da cuenta de esta situación: el 100\% de las personas que trabajan para Zolvers (plataforma de servicio enfocada en el trabajo de casas particulares) son mujeres; en AirBnb, ellas son el 56.7\%; en Freelancer y Workana, son el 32,8\% (donde la mayoría es asignación de microtareas a profesionales); en Mercado Libre, el $31 \%$, para luego caer estrepitosamente a niveles menores al $6 \%$ 
en plataformas más visibles como transporte de pasajeros, reparto y delivery en las calles (Rappi, Glovo etc.). Con la pandemia se ha incrementado la presencia de mujeres en plataformas y apps que ofertan trabajos de cuidados domiciliarios, principalmente de adultos mayores y personas con discapacidad (Partenio, 2021). En referencia a las plataformas de delivery, el confinamiento ha llevado a la "romantización de la cuarentena" con entregas de productos vendidos a través de apps, multiplicando las ganancias de las empresas e incrementando los niveles de desprotección de sus repartidores (Hidalgo Cordero et al., 2020).

De acuerdo al estudio de la OIT (2019, p. 15), las plataformas digitales de trabajo han significado uno de los cambios más importantes de la última década en el mundo laboral. Según la distinción establecida, se clasifican en dos tipos de plataformas: por un lado, las plataformas que están en línea y "el trabajo se terceriza mediante convocatorias abiertas a una audiencia geográficamente dispersa (una modalidad también conocida como crowdwork)"; por otro, "las aplicaciones (o apps) móviles con geolocalización, en las que el trabajo se asigna a individuos situados en zonas geográficas específicas". En este sentido, nuestro estudio se concentra en analizar la presencia mayoritaria de mujeres en plataformas de trabajo y en la posible combinación y/o presencia en plataformas digitales que no son de trabajo (tales como Facebook, Instagram, WhatsApp, Tiendanube etc.) pero que les permiten generar ingresos u ofrecer la producción de bienes y servicios en la web.

\section{Sector servicios: el crecimiento de la oferta de profesionales} en la web

Las mujeres profesionales que ofrecen sus servicios vía web se encontraban en ascenso antes de la pandemia. La creación de empresas o startups de personas que ofrecen servicios profesionales vía web se ha extendido en diferentes áreas como la comunicación, la arquitectura, la 
contaduría, el diseño gráfico, el asesoramiento legal y jurídico, la atención psicológica, la telemedicina y los servicios de cuidado (Partenio, 2021). Un sector en el cual nos queremos detener en detalle es el de las plataformas de trabajo dedicadas a la asignación de microtareas.

Conforme los testimonios de las entrevistas, es importante señalar que previo a la pandemia, había una situación menos diferenciada entre varones y mujeres profesionales, principalmente debido a la posibilidad de contar con redes y oferta de cuidado que permitían garantizar estas tareas. Sin embargo, una diferencia en este punto puede encontrarse en la cantidad de horas que comprendía la jornada laboral para trabajadores y trabajadoras, siendo jornadas más extensas las asumidas por las mujeres. En estas empresas de servicios ha prosperado el discurso y la promesa del "sueño de ser tu propio jefe", o jefa, y es aquí donde han prosperado los "emprendimientos", muchas veces para "conciliar" vida profesional con vida personal y/o con el sueño de libertad e independencia económica.

La popularización de estas modalidades laborales se extiende acompañada de un discurso que señala a la tecnología como generadora de una nueva oleada de "emprendedores", como parte de formas atípicas de empleo, al que se puede aspirar y que se encuentra lejos de las "rigideces" de tener un solo empleador, un horario fijo, un lugar donde ir todos los días a trabajar.

En este sector también podría enmarcarse emprendedores que han logrado armarse una carrera profesional autónoma exponiendo ideas originales en YouTube o Instagram. En este caso se trata de chefs y cocineras, humoristas y actores de stand up, mentores e influencers, charlas motivacionales etc. (Herrero Ruiz; Navarro-Beltrá, 2021). Estos modelos de presentación de ideas y consejos han logrado un grado de éxito tal que han alcanzado más reconocimiento, seguidores y sponsors que muchos artistas tradicionales; en algunos casos, reforzando estereotipos sexistas en modas y estilos de consumo (Martín García; Martínez Solana, 2019). Las nuevas formas de comunicación son vías sin mediadores para que el "talento" 
llegue a las personas sin pasar por managers y contratos comerciales y/o laborales (Barba Garrido, 2018).

También es posible encontrar profesionales que se rebuscan nuevas modalidades de generación de ingresos ofreciendo en solitario servicios por web (por ejemplo, contables, asesorías en administración de empresas, investigación y desarrollo, diseño etc.). En muchos casos, se trata de mujeres que se ven forzadas a renunciar a sus empleos formales en pos de combinar la actividad laboral con la reproducción social y combinar dos mundos que parecen irreconciliables. Dichas trabajadoras terminan optando por una forma más precaria de trabajo solo por mantener un ejercicio profesional y, al mismo tiempo, hacerse cargo del sostenimiento y cuidado de sus hijas e hijos, en muchos casos como cabezas de familia.

Con un desarrollo creciente - y potenciado por la pandemia -, la educación a distancia es un sector heterogéneo porque comprende diferentes niveles educativos y modalidades de la educación formal y no formal para todas las edades. Previamente a que se desatara la pandemia, el auge de los cursos online y las plataformas de educación a distancia incorporaban a más trabajadores en este tipo de empleos. Estos nuevos empleos eran mayormente tomados por mujeres profesionales jóvenes, ya que requieren habilidades y competencias en manejo de TICs, las jornadas de trabajo requieren de disponibilidad horaria, y las coberturas de protección son escasas. Este sector está creciendo fuertemente a medida que aumentan las demandas de capacitación con el cambio tecnológico en los puestos de trabajo y la virtualización de carreras está a la orden del día en la mayoría de las universidades (El Jaber, 2019). Durante la pandemia se inauguró una serie de debates y reflexiones sobre la extensión de la educación a distancia (El Jaber, 2021). Será cuestión de tiempo verificar cuanto de esta modalidad de trabajo de docencia a distancia quedará, una vez terminada la pandemia. Asimismo, los debates que se han dado en los sindicatos docentes con respecto a la intensificación del trabajo docente en pandemia, pueden ser una llave para profundizar las discusiones en torno a quienes 
realizan estas tareas desde plataformas de educación a distancia y no se encuentran sindicalizados. Lo cierto es que, sobre todo en educación superior, este nuevo paradigma ha comenzado a tener un mercado propio.

\section{Mujeres en la venta de bienes muebles, producción y servicios vía web}

Con la emergencia de la pandemia, las plataformas digitales han ganado mercado a nivel global. El modelo de negocios impulsado por Amazon es la máxima expresión. En Latinoamérica, hay empresas "unicornios" - nacidas en Argentina - que han ganado mercado; las que despegaron con "éxito" y ya cotizaban en bolsa de valores antes de la pandemia, han crecido a pasos agigantados. A su vez, muchas mujeres comenzaron a vender productos a través de redes sociales o se insertaron en plataformas de comercio minorista para localizar su producción en el mercado y poder subsistir. En la región, el auge de Mercado Libre fue descomunal ya que solamente en Argentina, aproximadamente el 50\% del comercio electrónico que se realizó durante la fase de ASPO, fue a través de esta empresa ${ }^{5}$.

Dentro de esta categoría se encuentra a personas no profesionales que deciden salir a vender bienes y servicios a través de las posibilidades que les ofrece la web. En cuanto a los productos, se trata de una fabricación propia (puede o no ser artesanal) y en otros casos se trata de personas intermediarias que hacen reventa online. ¿Qué posibilidades y opciones encuentran las mujeres aquí?

Es importante aclarar que este estudio pretendió mapear a mujeres que también combinan el uso de plataformas digitales que no son de trabajo como Facebook, Instagram, WhatsApp etc. -, para generar ingresos frente a la ausencia de otras posibilidades laborales. El fenómeno de los stores de Facebook es una realidad tan notoria que no hace mucho tiempo esta plataforma lanzó una nueva herramienta conocida como marketplace,

${ }^{5}$ Ver: https://www.cronista.com/apertura-negocio/empresas/MercadoLibre-gano3-5-veces-mas-en-la-cuarentena-las-razones-del-impulso-20200810-0002.html\# ga $=2.78203415 .222176682 .1602545979-1131210817.1602124764$ 
que expone aún más a quienes venden y ayuda a que se encuentren con les consumidores.

Entre las mujeres entrevistadas, es interesante mencionar que un sector de ellas puede reconocerse como "comerciantes" o no, pero todas utilizan internet como una vidriera a la calle capaz de promocionar sus productos a fin de lograr más exposición y nivel de ventas. En este caso, cuentan con motores de exposición como Facebook e Instagram, redes sociales donde ellas pueden ofrecer su producción, y hasta evaluar el pago de publicidad para llegar a más personas y potenciar el negocio. En algunos casos, los andamiajes que sostienen estas ventas online son precarios, los circuitos de respuesta con el usuario-consumidor, el despacho y entrega se sostienen a través de fases más artesanales, y muchas veces es asumido por alianzas de mujeres de la misma familia o del barrio. Para muchas de ellas, esta ha sido la única salida en el marco de la pandemia para sostener sus hogares frente a la caída o pérdida de ingresos de empleos informales.

Dentro del sector de ventas vía web también se puede encontrar a quienes se autoperciben como "emprendedores" y "emprendedoras", ya que, al igual que la categoría anterior, tratan de fijar sus propias reglas y estrategias de negocios en la plataformas web. Se trata de "microempresas", es decir, de mujeres que se reconocen "emprendiendo" ese comercio online y que lograron tener un local a la "calle" sin tener que pagar alquiler, utilizando una marquesina virtual. En esta categoría, las mujeres se insertan mayoritariamente en la venta de productos de belleza y cuidado personal, artesanías, indumentaria (muchas veces fabricada por ellas mismas), cosmética, higiene y otros (Viadana et al., 2016). Los varones se encuentran con mayor presencia en los rubros de productos tecnológicos, rodados, autopartes, elementos deportivos y demás. En este sentido, estudios recientes en la región señalan que hay una división sexual del trabajo que se replica en el mundo online (Cancela Allío, 2020). En el caso de las entrevistadas, ellas deciden el margen de tiempo que van a dedicarle a su jornada y arman una estrategia de ventas. En cuanto a los medios de pago, fijan las vías y modos de intermediación financiera, determinan las formas y tiempos de 
entrega, pueden armar promociones y descuentos, tienen libertad al elegir clientes y responder consultas; no tienen restricciones de cómo mostrarán su trabajo o productos (aunque la forma de presentación en las redes sea clave). También deciden cuánta exposición o publicidad quieren tener basándose en su capacidad e interés en reinvertir en el negocio.

Según las entrevistas realizadas, en este sector, las motivaciones para montar un comercio por la web son diferentes a quienes tienen una profesión. En estos casos se trata de: complementar ingresos existentes; lograr más exposición a un negocio que ya existía o que tuvo que cerrar sus puertas por la caída de la actividad en pandemia. En el caso de las mujeres, se enfatiza la posibilidad de "conciliar" el trabajo con la vida personal y familiar, sin tener que "moverse de casa". En el marco de la pandemia, uno de los desafíos más significativos que asumieron fue familiarizarse con la logística e infraestructura del comercio electrónico, como el crecimiento de los pagos y créditos a través de las fintech.

En términos de diferencias, se comienza a hacer visible la capacidad exportadora de los grupos (según diferencias de clase y género) y, por ende, el poder de alcanzar nuevos mercados. En el caso de las mujeres, en general se ubican en el sector servicios de la economía latinoamericana y, dentro de este sector, en los rubros menos exportables. Según el Banco Mundial (2019), el 80\% de las personas que trabajan en el sector servicios para la región son mujeres. Aquí comienzan a aparecer sesgos respecto de una realidad y otra que viven varones y mujeres en la red a la hora de encontrar un modo de subsistencia. Una línea a explorar, sobre todo en el contexto de la pandemia, es la extensión de modalidades de pago online, tarjetas y de billeteras electrónicas que, muchas veces, habilitan créditos; en estos casos, están siendo las mujeres las que se endeudan más para garantizar el emprendimiento y sostener a sus familias.

Otro punto clave a resaltar en términos de diferencias de género se refiere a los sistemas de calificación, dado que todo está parametrizado por la plataforma y el algoritmo juzga de manera automática el desempeño de les "usuarios-proveedores" en los tiempos y plazos esperados. Para 
evitar las calificaciones negativas y el impacto en su "reputación online", las entrevistadas mencionaban los malabares que hacen para sostener los intercambios desde sus hogares: "no te podés retrasar con los despachos, aunque estés con las cosas de la casa", "si se me enferma mi hijo, no puedo estar conectada respondiendo". Son precisamente las mujeres las que ven afectado su nivel de ventas porque el algoritmo no tendrá en cuenta si debe cuidar de una niña o debe garantizar las precondiciones del cuidado (compra de alimentos, gestiones del hogar etc.). A pesar del optimismo que se intenta instalar en torno al comercio electrónico, vemos que muchas de estas plataformas - que no son de trabajo - se sirven de la disponibilidad de tiempo de $24 \times 7$, de miles de usuarias y usuarios que intentan vender sus bienes y servicios, evitando regulaciones fiscales.

\section{Derechos en disputa en el marco de la pandemia}

Sin lugar a dudas, las categorizaciones de los nuevos tipos de empleos digitales y el impacto diferencial que tienen sobre las mujeres nos deja ver una realidad palpable y evidente: los mayores motivos por los que las trabajadoras - enmarcadas en este estudio - optan por estas modalidades, sea a través de plataformas, enmarcadas en el cuentapropismo o como "emprendedoras", se vinculan no sólo a las escasas posibilidades de creación de empleos formales sino también a la ausencia de políticas de corresponsabilidad de los cuidados y a la necesidad de tener que "conciliar" el trabajo productivo con el reproductivo ${ }^{6}$. A su vez, esta apuesta por la "conciliación" se apoya en la idea de poder elegir horarios y compatibilizar las tareas cotidianas, pudiendo trabajar entre medio de las interrupciones que implican sostener el trabajo de cuidados. Sin embargo, en el marco de la pandemia, la hiperconectividad ha afectado a trabajadores en general, y a las mujeres en particular. En las entrevistas realizadas en la etapa de ASPO, tanto las profesionales como las que comercializaban productos online reconocieron trabajar en horarios nocturnos o en aquellos momentos

${ }^{6}$ Se puede consultar una de las encuestas realizadas en este contexto: https:// lasargentinastrabajamos.com/publicaciones\# 
donde la familia descansaba, para poder contar con el espacio y tiempo para cumplir con entregas y preparación de pedidos.

Estas modalidades creadas a través de las plataformas analizadas acarrean nuevas formas de precariedad laboral. Lo que se vislumbra de fondo es la necesidad de conquistar derechos, sobre todo para las mujeres y personas trans. Estos nuevos derechos pueden revitalizar viejos debates que ya estaban presentes en la agenda feminista - como el reconocimiento de los cuidados como trabajo - y en la agenda sindical - como el debate por la intromisión del trabajo remunerado en la vida de trabajadores a través de las TICs, mucho antes de que las plataformas de trabajo se extendieran en el Norte y el Sur globales.

Una de estas disputas en el marco de la pandemia se ha dado en el debate $^{7}$ por la desconexión. En el caso del derecho a la desconexión digital, su definición está signada por las nuevas enfermedades laborales tipificadas por la OMS en el año 2019². En efecto, el surgimiento del síndrome de agotamiento crónico llevó a diversos países a comenzar a legislar sobre este derecho. En el caso de la UE, Francia fue pionera en esta materia en el año 2016, alcanzando al sector de empleo formal. En el 2017, Italia debatió este derecho en una ley de "trabajo inteligente", en que es posible diferenciar tiempos de descanso y las medidas técnicas y organizativas necesarias para garantizar la desconexión. Posteriormente se dan las discusiones en Bélgica, Canadá, Filipinas, India, España, Canadá, EEUU y Portugal. En el caso de Alemania, se impulsaron deliberaciones sobre el derecho a la desconexión en el marco de la negociación colectiva por empresa (Scasserra, 2020).

Muchas veces se piensa que el derecho a desconexión es el derecho que tiene cada persona a apagar sus dispositivos digitales. Si bien esto es cierto, es mucho más que eso. Es el derecho que tiene cada trabajadora a apagar sus dispositivos, a no responder mensajes ni a recibirlos fuera del horario de trabajo. Esto es así porque desde el momento en que se

${ }^{7}$ Consultar: https://uniglobalunion.org/sites/default/files/imce/right to disconnect-es.pdf ${ }^{8}$ Ver la declaración de la Organización Mundial de la Salud sobre el síndrome del Burnout (o síndrome de agotamiento) en su Clasificación Estadística Internacional de enfermedades y problemas relacionados a la salud https://www.who.int/news/item/28-05-2019-burn-outan-occupational-phenomenon-international-classification-of-diseases 
ve fusionada la vida laboral y personal en los dispositivos electrónicos, se pierde la capacidad de apagarlos, porque esto significaba una pérdida de conexión con vínculos familiares y sociales.

Lo cierto es que la labor vía plataformas de trabajo comenzó a imponerse como modalidad bajo una narrativa riesgosa para la protección de les trabajadores. La narrativa enaltece la facilidad para acceder a una oportunidad laboral y, en el caso de los servicios profesionales, se plantea la oportunidad de competir - como lo mencionó el responsable de una plataforma - y de "internacionalizar el trabajo individual". Las plataformas ofrecen esa ilusión de poder elegir horarios laborales, y no ser molestada fuera de la jornada elegida. De hecho, esta es la gran excusa que utilizan las empresas para afirmar que sus "colaboradores" no son trabajadores en relación de dependencia sino "emprendedores" o independientes. Esto es doblemente preocupante para las trabajadoras de estas plataformas ya que no pueden reclamar pausas, interrupciones de la jornada laboral ni tiempo ni licencias que permitan garantizar el derecho al cuidado de personas dependientes en el hogar.

De la misma manera, estas modalidades de conexión las 24hs, también se han extendido en otros sectores de servicios, incluso en las relaciones laborales en el sector público. El vínculo entre la disponibilidad de tiempo y la evaluación de desempeño se juegan en otro terreno: si antes era un "jefe de recursos humanos", ahora son las mismas plataformas las que pueden penalizar si se rechaza pedidos o no se responde consultas online. El largo año de pandemia ha extendido estas modalidades, que incluyen no solo vínculos laborales, sino también sociales, comerciales, educativos. Bajo estas dinámicas laborales que nos exigen estar disponibles las 24hs. y los 365 días del año, la duración de la jornada de trabajo es fundamental. El derecho a la desconexión digital ${ }^{9}$, en este sentido, es clave en esta nueva agenda.

Hemos podido ver como las nuevas tecnologías irrumpieron en un mundo que aún no había saldado el debate sobre el trabajo doméstico $y$, dadas las condiciones del mercado laboral, provocaron una oleada de

9 Para analizar los alcances de este derecho en el marco de la ley de teletrabajo en Argentina, ver la ley y su reglamentación: https://www.argentina.gob.ar/justicia/derechofacil/leysimple/teletrabajo 
mujeres que se encuentran ante la opción de poder "conciliar" ambos mundos a costa de sus derechos laborales, encasillándolas una vez más en su rol social de reproductoras de la vida. A falta de políticas integrales que repiensen la injusta organización social del cuidado ${ }^{\mathbf{1 0}}$, la incorporación de las trabajadoras en estas plataformas inaugura desafíos para la organización sindical y para la agenda feminista.

En el caso de Argentina, las demandas y acciones del feminismo se han concentrado en problematizar el encierro doméstico de las mujeres, en generar condiciones de acceso al trabajo remunerado con tiempo para el desarrollo de su vida profesional, su carrera, su vocación, y poco a poco empezar a reconocer y redistribuir el cuidado de la familia. El impulso de la agenda de los cuidados ha cobrado fuerza en los últimos años en las trabajadoras de las bases sindicales que se identifican con los feminismos. Las movilizaciones internacionales del $8 \mathrm{M}$ y la fuerza del movimiento feminista argentino ha sido clave para movilizar estas agendas en torno al trabajo.

La capacidad de elegir horarios parece ser el gran tema en cuestión, ergo es necesario obtener este derecho para más trabajadores, a fin de que sea indiscutida la relación de dependencia existente entre las plataformas y sus trabajadores. Esto abrirá la puerta para regular las plataformas de trabajo y las que se presentan como meras intermediarias de ventas online.

\section{Implicancias para la organización colectiva en tiempos de economía digital}

Las demandas del movimiento feminista latinoamericano - que ha puesto en el centro de la escena las luchas contra la violencia, el derecho a decidir y la valorización del trabajo de las mujeres - llega hasta los oídos de una trabajadora aislada entre las paredes de su hogar. Lo cierto es que

10 Por ejemplo, nos referimos al diseño de un Sistema Integrado de Cuidados como en el caso de Uruguay. También nos referimos al diseño de políticas que combinen la ampliación de los tiempos para cuidar (licencias remuneradas) con el reconocimiento del cuidado como derecho; la inversión en infraestructura pública del cuidado es clave para ampliar la oferta. 
se presentan nuevos y complejos desafíos respecto a las posibilidades de regulación laboral y protección social, más aún en contexto de aislamiento social por la pandemia. Las condiciones de precariedad que moldean la situación de estas trabajadoras requerirán de un esfuerzo extra por parte de los actores que integran el mundo del trabajo. En este escenario, es urgente que nuestra agenda feminista por la justicia digital debata sobre los bienes comunes de datos (Sai; Scasserra, 2020), la protección y potestad de los datos que generamos a diario de manera individual o colectiva. De la misma manera, es vital impulsar la consigna de que todo trabajo que genere datos debe estar vinculado a derechos digitales.

Desde la narrativa que estimula la idea de convertirse en "tu propia jefa", los medios de comunicación masivos emiten mensajes que enaltecen las ventajas de ser "emprendedora", en lugar de incentivar los sentidos que se asocian con la condición de clase trabajadora. La publicidad y el mensaje de las empresas tiende a crear una conciencia en cada trabajador o trabajadora que la hace autopercibirse como artífice y/o "líder" de su proyecto, como perteneciendo al sector empleador. No solo eso, sino que, además, los procesos algorítmicos que seleccionan la información que vemos en redes sociales tienden a encasillar al individuo dentro de lo que considera cercano en sus preferencias. Está comprobado que los algoritmos muestran aquello que nos parece aceptable o que encuadra con nuestra opinión ya formada de la realidad (González, 2019). Asimismo, las noticias falsas que circulan, las propagandas y espacios pagos en redes, así como también los trolls y falsos trending topics representan sesgos difíciles de sortear a la hora de generar opinión y sentido crítico.

Así surge la subsiguiente pregunta: ¿cómo lograr la participación activa de las trabajadoras de plataformas en el movimiento sindical? Aquí hay un doble efecto - por un lado, el trabajo externalizado y remoto no suele estar bajo las esferas de representación sindical; por otro lado, es difícil que una trabajadora o trabajador se acerque voluntariamente si no se percibe como trabajador. El peligro de la pérdida de representatividad, 
vaciando a las estructuras sindicales de delegadas mujeres debido a que permanecen invisibles en las plataformas digitales, resulta en una amenaza para el movimiento sindical internacional. Por ende, una primera conclusión puede arrojarse aquí: es necesario comenzar a elaborar estrategias de comunicación y representación sindical para estas trabajadoras. Prueba de ello son las formas de organización 4.0 que se están planteando en otros países (Niebler; Kern, 2020). También puede verse el caso de la organización sindical de trabajadores de plataformas de delivery en Argentina (Diana Menéndez et al., 2020; Del Bono, 2020).

Es menester, desde las organizaciones sindicales, empezar a generar estrategias no sólo desde la afiliación sino también desde la capacitación: la construcción de una nueva generación de representantes sindicales en trabajo remoto necesitará un esfuerzo extra en términos de formación sindical. Las estrategias de formación en línea representan una oportunidad única para lograr llegar a esa trabajadora y darle herramientas. Estas estrategias comenzaron a articularse en el marco de la pandemia en algunos sindicatos de servicios, pero deben ser aún exploradas y diagramadas por las organizaciones sindicales en Argentina.

En el contexto previo al Covid-19, la explotación y la sobrecarga de trabajo - remunerado y no remunerado -, acompañado de la mano de la tecnología, logró armar un esquema de trabajo vía plataformas sumamente "eficiente", y que ya dificultaba el tejido de solidaridad sindical. Asimismo, las prácticas de militancia conocidas, las formas de compañerismo y solidaridad se forjaban con encuentros cara a cara. Sin embargo, el contexto de Covid-19 puso en jaque muchas de estas prácticas y estilos de organización y movilización dentro de las militancias (sean sindicales o de otro tipo). Pensando en la profundización y extensión tanto del empleo remoto y del trabajo a través de una plataforma digital, probablemente sea necesario comenzar a elaborar una nueva estrategia de representantes sindicales que "caminen" - en términos virtuales "las casas", reemplazando la figura del viejo delegado sindical que "caminaba las fábricas". 
Pero en el marco de la pandemia, es preciso repensar no solo las estrategias sindicales frente a la virtualización, sino cuáles son las disputas por los nuevos derechos, complejizando la mirada sobre la presencia de trabajadoras en distintas plataformas como las mencionadas en este estudio. Una estrategia de comunicación, afiliación y formación virtual es sumamente necesaria, pero con eso solo no alcanza, hay que salir al encuentro de esa trabajadora y hacerla sentir parte de una organización que estará presente para ella de manera remota, y de manera física si así lo precisara y si el contexto post-Covid lo habilita. La inteligencia artificial no ha logrado crear, al día de la fecha, empatía, amor y solidaridad artificial. La conjunción de estrategias puede ser la llave para lograr una nueva generación de delegadas remotas, que participen activamente desde sus hogares en la política y la ampliación de la sindicalización, tejiendo redes de solidaridad y confianza, logrando un movimiento sindical en la web, presente y activo. Esta llave es clave a la hora de abordar la violencia machista y el acoso laboral en entornos virtuales que pueden padecer las trabajadoras "aisladas" en sus casas y por parte de sus jefes o "colaboradores". Hay aquí algunos claves para reflexionar sobre los puntos de contacto entre teletrabajadoras alcanzadas por la reciente normativa y quienes aún continúan trabajando en plataformas que solo las reconocen como "contratistas independientes", "colaboradoras" o "emprendedoras".

En este sentido, la reciente Ley de Teletrabajo en Argentina ${ }^{11}$ no solo establece la capacidad de las personas trabajadoras de elegir horarios compatibles con las tareas de cuidados, sino que les otorgó el derecho a la desconexión digital y el derecho a la reversibilidad. En efecto, la reversibilidad implica que quien haya elegido, por algún motivo, teletrabajar $y$, en un futuro, descubra que no es lo que desea, pueda volver a trabajar en modalidad presencial. A su vez, la desconexión digital posibilita organizar los tiempos y la disponibilidad para las responsabilidades de cuidados. En

11 Dicha ley estipula su implementación luego de 90 días contados a partir de la finalización del Aislamiento Social, Preventivo y Obligatorio (ASPO). 
este punto, serán claves las políticas de corresponsabilidad de los cuidados que se diseñen entre Estado, mercado y hogares. Una trabajadora que no es constantemente molestada fuera de su jornada tiene la potestad de poder organizarse mejor, dividir tareas de una manera más equitativa, incluso destinar tiempo al autocuidado.

La Ley de Plataformas que está actualmente en discusión en el país, va en consonancia con esta, manteniendo el derecho a la desconexión digital, y permitiendo a trabajadores y trabajadoras elegir su horario sin ir en detrimento de sus derechos sindicales y laborales.

En este sentido, los desafíos 4.0 que ya se han presentado para los sindicatos, interpelan sobre las formas que asumirá esa organización colectiva como punto de encuentro, como reunión social, como espacio de apoyo y generación de sentido de pertenencia para la nueva camada de trabajadoras que están en sus hogares. Presentamos a continuación algunos apuntes para complejizar la articulación entre la agenda sindical y feminista.

- La estrategia destinada a trabajadoras de las plataformas de trabajo que están en sus hogares debería ser una que unifique varias vías de acción. Por un lado, invertir en una eficiente estrategia de comunicación sindical/social, con presencia activa en la red, con especialistas en el área para diseñar campañas con lemas simples pero que interpelen la realidad de esas trabajadoras. Por otro lado, invertir en la maximización de ese mensaje en las redes, que "salga" a buscar a esa trabajadora que está en su casa tratando de generar ingresos y cuidando a su familia. No alcanza con crear una página y dejarla ahí, el Big Data también puede ser puesto al servicio de la búsqueda de esa trabajadora. Hoy por hoy se venden los espacios de publicidad en Facebook, por mencionar alguno, donde la empresa tiene enorme cantidad de datos de sus usuarios y va segmentando de acuerdo a perfiles, aquellos que son afines a mirar con agrado nuestro mensaje o publicidad. 
- Invertir en una estrategia de formación político/sindical en línea, con plataformas atractivas y dinámicas para facilitar la tarea de formación a esas trabajadoras mujeres y personas LGBT $+{ }^{12}$. Explorar las modalidades híbridas o semi-presenciales que permitan combinar escenarios en las etapas de distanciamiento social.

- Considerando un escenario post-covid, conformar un nuevo cuerpo de representantes sindicales que salgan al encuentro de esas trabajadoras y realicen visitas a los hogares explicándoles los beneficios de afiliarse y mostrándoles que muchas otras comparten su realidad y sus reclamos. Esto requiere un esfuerzo extra no explorado por muchas organizaciones.

- Contar con un lugar al que recurrir: un punto de encuentro y reunión para socializar cuando esa trabajadora pueda o quiera plantear una problemática. Explorar puntos en encuentro en sedes sindicales virtuales $y / 0$ presenciales.

- Crear redes de contención en espacios virtuales entre trabajadores. Grupos de chats o canales de difusión, pueden ser herramientas eficaces para que la comunicación sea constante y recree un "ambiente de trabajo" con colegas que comparten su realidad a pesar de no estar en contacto o jamás haberse visto cara a cara.

- Ganar el derecho a la desconexión digital para todas las personas trabajadoras, a fin de que las plataformas dejen de ser una opción solo por el hecho de permitir flexibilidad a un esquema de horarios y evite que más mujeres se vuelquen a estos por dicho motivo, precarizando aún más la situación de ellas en el futuro.

\footnotetext{
12 Remarcamos en este punto las estrategias de formación desplegadas por las organizaciones del colectivo trans y travesti en Argentina, por ejemplo, la plataforma de ATTTA, los ciclos de formación de Transistemas. Estas estrategias de inclusión digital y sociolaboral cobran mayor relevancia en el marco de los desafíos que inaugura la reciente aprobación de la Ley de Cupo Laboral Trans y Travesti.
} 
Estas son solo algunas propuestas para pensar y discutir en base a las realidades sindicales y sociales de cada país, que pueden servir como insumos para elaborar una nueva estrategia de acercamiento a estas trabajadoras aisladas. El trabajo en esa dirección es necesario para comenzar a representar una masa de trabajadores a distancia (tanto varones como mujeres cis, lesbianas, no binaries, travestis y trans) que viene creciendo en los últimos años. Como expresamos anteriormente, en las mujeres cobra una relevancia adicional debido a la opción que toman por estas plataformas para "conciliar" la producción y reproducción de la vida en su doble jornada laboral. De lo contrario, las trabajadoras perderán poco a poco representación en las estructuras sindicales y sus demandas se verán desdibujadas desde el movimiento sindical.

Sofia Scasserra es licenciada en Economía (UCA) e investigadora y docente del Instituto del Mundo del Trabajo Julio Godio, Universidad Nacional de Tres de Febrero, Argentina.

\sscasserra@untref.edu.ar

Flora Partenio es Doctora en Ciencias Sociales (UBA), directora de la Licenciatura en Relaciones del Trabajo de la Universidad Nacional Arturo Jauretche y docente de posgrado (UNGS/UNSJ/UNMdP), Argentina. Integra la Red de feministas DAWN.

$\triangle$ fpartenio@unaj.edu.ar 


\section{Referencias}

1. ATTTA - Asociación de Travestis, Transexuales y Trangéneros de Argentina. Orgullose en el trabajo, Empleadores. (Sitio institucional). Buenos Aires. 2020. Disponible en: http://attta.org.ar/category/empleadores/

2. BANCO MUNDIAL. Employment in services, female (\% of female employment). World Bank Data, 2019. Disponible en: https://data.worldbank.org/indicator/ SL.SRV.EMPL.FE.ZS?view= chart

3. BARBA GARRIDO, Juan Carlos. Agencias de publicidad e influencers, ¿amigos o enemigos? analizando la relación entre el contenido de campaña, instagram e influencers. 2018. Tesis. (Maestría en Comunicación con Mención en Comunicación Digital). Universidad Casa Grande, Guayaquil, Ecuador, 2018.

4. CANCELA ALLÍO, Victoria. Internet ireproductor de la división sexual del trabajo en el mundo on line? Estudio cuantitativo sobre el trabajo productivo y reproductivo en los hogares uruguayos. In: RIVOIR, Ana Laura (coord.). Tecnologías digitales y transformaciones sociales: desigualdades y desafíos en el contexto latinoamericano actual. Buenos Aires, Montevideo: CLACSOUDELAR-Observatic, 2020. p. 229-245.

5. CARRASCO, Cristina (ed.). Mujeres y Economía. Madrid: Icaria Editorial. 1999.

6. CEPAL - Comisión Económica para América Latina y el Caribe. La pandemia del COVID-19 profundiza la crisis de los cuidados en América Latina y el Caribe. Informes Covid-19. Santiago: CEPAL, abril, 2020.

7. COOK, Cody; DIAMOND, Rebecca; HALL, Jonathan V.; LIST, John A.; OYER, Paul. The gender earnings gap in the gig economy: evidence from over a million rideshare drivers. Working Paper, n. 3637, Stanford University, May 2020. Disponible en: https://web.stanford.edu/ diamondr/UberPayGap.pdf

8. DALLA COSTA, Mariarosa. Dinero, perlas y flores en la reproducción feminista. Madrid: Akal. 2009.

9. DEL BONO, Andrea. Nuevas tecnologías y relaciones laborales: la gestión algorítmica y su impacto sobre los trabajadores de plataformas. Voces en el Fénix, 80, p. 86-91, julio 2020.

10. DIANA MENÉNDEZ, Nicolás; ARIAS, Cora Cecilia; HAIDAR, Julieta. ¿Sindicalismo 4.0? La organización de trabajadores de plataformas en Argentina. Sociología del Trabajo, n. 97, p. 59-69. https://doi.org/10.5209/stra.71907

11. FEDERICI, Silvia. El patriarcado del salario. Críticas feministas al marxismo, Madrid: Traficantes de Sueños. 2018a.

12. FEDERICl, Silvia. Conferencia Inaugural con motivo de la entrega del Honoris Causa, Mendoza: Universidad Nacional de Cuyo, octubre, 2018b. Disponible en: https://www.youtube.com/watch?v=eE ISFpAvjQ 
13. EL JABER, Grisel. Trabajador/a virtual. Implicancias de ser un trabajador a distancia. In: IV Jornada Educación a distancia y universidad. Panel 3. "Trabajador/a Virtual": implicancias de ser un trabajador/a de educación a distancia, 27 de septiembre, Buenos Aires: FLACSO Argentina. 2019. Disponible en: https://www.youtube.com/watch?v=00902Miu9Rk

14. EL JABER, Grisel (comp.). Actas de V Jornadas de Educación a Distancia y Universidad. 24, 25, 26 y 27 de noviembre de 2020. Ciudad Autónoma de Buenos Aires: Facultad Latinoamericana de Ciencias Sociales - FLACSO Sede Argentina. 2021.

15. GASKELL, Adi. Are female Uber drivers paid less than male drivers? Forbes, 12 feb. 2018. Disponible en: https://www.forbes.com/sites/adigaskell/2018/02/12/ are-female-uber-drivers-paid-less-than-male-drivers/\#951056b56c60

16. GONZÁLEZ, Felipe. Big data, algoritmos y política: las ciencias sociales en la era de las redes digitales. Cinta Moebio, n 65, p. 267-280, 2019.

17. HAWKINS, Andrew. Female Uber drivers earn \$1.24 per hour less than men: study. The Verge, 6 feb. 2018. Disponible en: https://www.theverge. com/2018/2/6/16980072/uber-driver-gender-earnings-gap-study

18. HERRERO RUÍZ, Laura; NAVARRO-BELTRÁ, Marián. Diferencias de género en la comunicación de las y los "influencers": el caso de los instagramers gastronómicos. Cuestiones de Género: de la Igualdad y la Diferencia, n. 16, p. 536-564, 2021.

19. HIDALGO CORDERO, Kruskaya; PARTENIO, Flora; SCASSERRA, Sofía; VALENCIA CASTRO, Belén. iCuerpos que no importan! delivery en tiempos de coronavirus. Medium, 28 abril, 2020. Disponible en: https://medium.com/ especial-ella-en-cuarentena/cuerpos-que-no-importan-delivery-en-tiempos-decoronavirus-8293744b283d

20. MADARIAGA, Javier; BUENADICHA, César; MOLINA, Erika; ERNST, Christoph. Economía de plataformas y empleo ¿Cómo es trabajar para una app en Argentina? Buenos Aires: CIPPEC-BID - OIT, 2019. Disponible en: https:// www.cippec.org/wp-content/uploads/2019/05/Como-es-trabajar-en-una-app-enArgentina-CIPPEC-BID-LAB-OIT.pdf

21. MARTíN GARCÍA, María T.; MARTíneZ SOLANA, María Y. Mujeres ilustradoras en Instagram: las influencers digitales más comprometidas con la igualdad de género en las redes sociales. Visual Review, International Visual Culture Review, v. 6, n. 2, p.59-68. 2019.

22. NIEBLER, Valentin; KERN, Annemarie. Organising YouTube: a novel case of platform worker organising. Trade Unions in Transformation 4.0. Berlín: FriedrichEbert-Stiftung, set., 2020.

23. OIT - Organización Internacional del Trabajo. Las plataformas digitales y el futuro del trabajo. Cómo fomentar el trabajo decente en el mundo digital, Ginebra: OIT, 2019. 
24. OMC - Organización Mundial del Comércio. Sesión 55 Mujeres y Comercio Electrónico: de los datos a la implementación, Genebra: OMC, 2019. Disponible en: https://www.wto.org/english/forums_e/public_forum18_e/pf18programme_e.htm

25. OTTAVIANO, Juan Manuel. Salven el trabajo asalariado. ¿Cuál es el pacto conocido que permitió mayores márgenes de protección social? El Cohete a la Luna, 26 abr. 2020. Disponible en: https://www.elcohetealaluna.com/salven-eltrabajo-asalariado/

26. PARTENIO, Flora. Ofensiva corporativa sobre los cuidados. (Ponencia, 2 junio 2021). Megaproyectos, herramienta corporativa al asalto de la vida (Webinar). Bilbao: OMAL, 2021

27. PARTENIO, Flora. Retrocesos 4.0: los desafíos de la protección social de les trabajadores de plataformas. Dawn, 12, marzo, 2020a. Disponible en: https:// dawnnet.org/2020/03/retrocesos-4-0-los-desafios-de-la-proteccion-social-de-lestrabajadores-de-plataformas/

28. PARTENIO, Flora. Virtualización y precarización de nuestros derechos laborales. Boletín de la AAIHMEG, v. 4, n. 2, Julio, 2020 b.

29. PARTENIO, Flora. La producción de géneros: experiencias de mujeres trabajadoras en la gestión de fábricas recuperadas. 2013. Tesis (Doctorado en Ciencias Sociales), Universidad de Buenos Aires, Buenos Aires, 2013.

30. PICCHIO, Antonella. Social reproduction: the political economy of the labour market. Cambridge: Cambridge University Press, 1992.

31. RODRÍGUEZ, Dinah; COOPER, Jennifer (comp.). Debate sobre el trabajo doméstico: antología. México: Universidad Nacional Autónoma de México, Instituto de Investigaciones Económicas, 2005.

32. RODRÍGUEZ ENRÍQUEZ, Corina; MARZONETTO, Gabriela. Organización social del cuidado y desigualdad: el déficit de políticas públicas de cuidado en Argentina. Revista Perspectivas de Políticas Públicas, v. 4, n. 8, p. 105-134, 2015. https://doi.org/10.18294/rppp.2015.949

33. SAI, Leonardo Fabián. Fragmentos de fragmentos: vida psíquica, forma estética, potencia histórica. Revista Espectros, v. 5, n. 6, 2019. Disponible en: http://espectros.com.ar/numero-6-fragmentos-de-fragmentos-vida-psiquicaforma-estetica-potencia-historica-leonardo-fabian-sai/

34. SCASSERRA, Sofía. Cuando el jefe se tomó el buque, el algoritmo toma el control. Buenos Aires: Fundación Foro del Sur, 2019. Disponible en: https:// lasargentinastrabajamos.com/publicaciones

35. SCASSERRA, Sofía; SAI, Leonardo Fabián. La cuestión de los datos, plusvalía de vida, bienes comunes y Estados inteligentes. Buenos Aires: Fundación Friedrich Ebert. Disponible en: http://www.fes.org.ar/public/LA\%20 CUESTI\%C3\%93N\%20DE\%20LOS\%20DATOS.pdf

36. SCASSERRA, Sofía. Derecho a la desconexión. Revista Anfibia, Buenos Aires, 2020. http://revistaanfibia.com/ensayo/derecho-la-desconexion/ 
37. SLEE, Tom. Lo tuyo es mío. Madrid: Taurus, 2016.

38. VACA TRIGO, Iliana. Oportunidades y desafíos para la autonomía de las mujeres en el futuro escenario del trabajo. Serie Asuntos de Género. Santiago de Chile: CEPAL, 2019.

39. VIADANA, Claudia A.; ZUBELDÍA, Maria Lourdes; TOMARELLI, Jimena; MORALES, Oscar. Mujeres emprendedoras: una aproximación empírica. In: Vigesimoprimeras Jornadas "Investigaciones en la Facultad" de Ciencias Económicas y Estadística. Actas... Noviembre, 2016.

40. VILLOTA, Paloma de (ed.). Globalización a qué precio: su impacto en las mujeres del Norte y del Sur. Madrid: Icaria Editorial. 2001.

41. ZUBOFF, Shoshana. The age of surveillance capitalism: the fight for a human future at the new frontier of power. New York: Public Affairs, 2019.

Recibido: 19 mar. 2021.

Aprobado: 05 ago. 2021. 